\title{
Design a Cost Optimum for 5g Mobile Cellular Network Footing on NFV and SDN
}

\author{
Bura Vijay Kumar, Yerrolla Chanti, Nagender Yamsani, Srinivas Aluvala, Bandi Bhaskar
}

\begin{abstract}
With the short development of patron traffic, management innovation, and the tenacious want to lessen prices, the existing portable administrators are seemed with a few problems. In structures administration, thoughts have advanced going for cost lower, increment of device adaptability and association adaptability, to be precise software program described networking (SDN) and community features virtualization (NFV). Network functions virtualization mitigates the reliance on tough product, where flexible device capacities are conveyed as programming digital device works on ware servers at cloud infrastructure, server farms. SDN offers a programmable and adaptable system control by means of decoupling the versatile system features into manipulate plane and records plane capacities. The shape of the reducing part flexible system (5G) needs new arrangement and dimensioning fashions to accomplish an ideal plan which supports extensive scope of site visitor's requests. We recommend 3 improvement models that go for limiting the machine burden cost simply as server farm belongings fee by means of finding the precise situation of the server farms too the SDN and NFV portable system capacities. The development arrangements show the exchange offs between the numerous server farm groups, i.e., centralized or circulated, and the numerous value elements, perfect machine burden cost or server farm property fee. We advocate a Pareto best multitarget model that accomplishes stability among device and server farm value. Moreover, we use earlier derivation, in view of the preparations of the unmarried objectives, to preselect server farm areas for the multi-target model that consequences in lessening the streamlining intricacy and accomplishes reserve price range in run time at the same time as preserving an insignificant optimality hole.
\end{abstract}

Keywords: Network Functions Virtualization (NFV), Software Defined Networking (SDN), 5G, versatile center system, advancement.

\section{INTRODUCTION}

Next era 5G calls for new thoughts and architectures for the transportable device a good way to improve the provided presentation, to amplify its sending adaptability and to reduce its expense. A simple part that forces a few problems to versatile directors is the transportable center system.

The cellular middle network is presently populous with a number of integrated hardware based totally network

Revised Manuscript Received on July 10, 2019.

Bura Vijay Kumar, Department of Computer Science and Engineering, S R Engineering College Warangal Urban, Telangana India

Yerrolla Chanti, Department of Computer Science and Engineering, S R Engineering College Warangal Urban, Telangana India (yerrollachanti308@gmail.com)

Nagender Yamsani, Department of Computer Science and Engineering, S R Engineering College Warangal Urban, Telangana India (nagendar.y@ srecwarangal.ac.in)

Srinivas Aluvala, Department of Computer Science and Engineering, $\mathrm{S} \quad \mathrm{R}$ Engineering College Warangal Urban, Telangana India (srinu.aluvala@gmail.com)

Bandi Bhaskar, Department of Computer Science and Engineering, S $\mathrm{R}$ Engineering College Warangal Urban, Telangana India (bandibhaskar1526@gmail.com) (vijaykumar.bura@gmail.com)

features. This edges the cellular middle network's reliable and scalability to address the severe growth in customers' traffic. This effects in extended arrangement cycles and boundaries the provision novelty and overall presentation development. An Alternative predicament in the modernday center community design is the disbursed control level layout which contributes to the provided overall performance to user and encourages rigidity toward the network formation. as a result, in keeping with these tests, the contemporary distribution induces a high entire cost of rights on operators to construct and function the cell core network and delays the novelty in the existing services by way of the cell network workers [1]. In the networking, major ideas are being measured for the middle network structure in this direction of the subsequent era 5G [2], specifically for the Network Functions Virtualization (NFV) and Software Defined Networking (SDN) [4]. Network Functions Virtualization leverages the ideas of information Technology virtualization capabilities, wherein capabilities may be executed in software and deployed as virtual system functions on service hardware at cloud, i.e., facts middle (DC) infrastructure. (NFV) Network Functions Virtualization gives extra elasticity through eliminating the need on the hardware and it authorize greater opportunities for small deployment cycles and provider improve. Hence, NFV (Network Functions Virtualization) is anticipated to lessen the value of portable networks. Software defined networking [5], disconnect the statistics and manipulates planes of system capabilities and presents an open application program interface, e.g., Open Flow Protocol (OPF) [6] such as a modern defector preferred, among the disconnected planes. The manage plane remains found out by software defined networking switches that constitute the Software defined network statistics plane to a cellular center network, whatever we mention to a software defined networking+ switches. Software defined networking+ controllers enforce distinct motive statistics plane capabilities, e.g., General Packet Radio Services Tunneling Protocol -GTP sapping that compresses customers' site visitors or accounting and charging capabilities. Software defined network gives a logical community, which streamlines the network process and manages. Additionally, software defined network In these paintings, we suggest three streamlining fashions that move for locating the appropriate plan for a transportable middle system depending on SDN and NFV. These fashions furnish perfect fee arrangements concerning the accompanying perspectives: a) the best scenario of the server farms, which

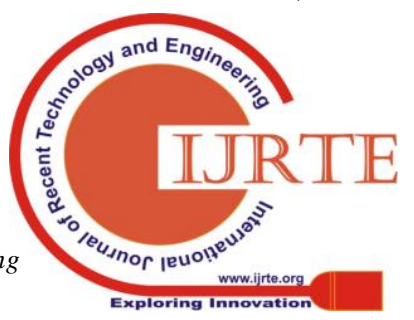


host the flexible Virtualization Network Function (VNF) and transportable software defined network controllers, b) Ideal plotting of Virtualization Network Function (VNFs) and switches to every datum focus, c) Wide variety in addition to function of the flexible particular cause software defined network+ controllers. The suggested development fashions reflect inactivity stipulations on behalf of the two manipulate and statistics planes. A vast assessment is accomplished, which produces distinctive viable ability anchors on the way to locate the suitable gadget plan that supports the normal huge scope of converting visitors in $5 \mathrm{G}$. Permits a centralized manage view that offers the operators with the chance to gain more effective community manage. Here are exceptional cost influences that may be better in the novel center machine configuration depending on (SDN) software defined network and (NFV) Network Functions Virtualization. The primary price element is the gadget burden, price which speaks to the fee of the system property anticipated to assist the information and control aircraft traffic of the transportable center system. In previous work [7], we provide the advancement ideal that consolidates each Network function virtualization and software defined network center machine capacities. Notwithstanding, we've got just considered the improvement of the system burden fee. We likewise centered uniquely round statistics aircraft function chains and records aircraft idleness necessities. Thus, on this work, we expand the gadget burden price development version to include manipulate plane capacities chains and Manage dormancy stipulations to offer a gradually some distance attaining with the aid of and big version for a transportable center system. The other fee issue that is presented via the thoughts of Network function virtualization and software defined networking is rate of the server farms foundation which has Network function virtualization and software defined network switches. We advocate any other advancement version for the server farm assets price to break down the alternate offs between the machine burden and server farm belongings price factors. Moreover, a multi-goal version is proposed if you want to find out Pareto perfect value arrangements thinking about each the system just as server farm property price. We moreover use earlier deduction, in view of the single goal preparations, to pre-pick out hopeful server farm regions for the Pareto perfect multi-target version that allows you to improve its run time [6]. Every one of the 3 proposed models bear in mind the facts and control aircraft dormancy as key execution measurements, simply as the quantity of server farms which are applied on behalf of sending. Moderation of the paper remains organized in place of pursues. Area two exhibits the outline of the inspiration and correlated reducing facet. In Segment (III), the layout of the versatile center device depending on has Network function virtualization and software defined network is presented through an examination on behalf of the statistics plus manipulate planes. Segment (IV) Introduction Dukes the numerical details plus methodologies to the suggested models. A wide assessment of the models is brought in Segments (V) and (VI). At ultimate, ends in addition to ventures on behalf of destiny paintings are delivered in Segment (VII).

\section{ASSOCIATED WORK}

Literature survey it can be classified into two regions. First location is troubled the structural designs and employment schemes for Software Define Network and Network function virtualization cellular essential networks. Another region studies the exhibiting and optimization of Network function virtualization or software defined networking, for cellular systems and outdated Internet Protocol Networks. With the both the regions, it must to observe a clean cut up of the effort into one or the other Network function virtualization and software defined networking related.

\section{A. Network function virtualization and software defined networking Portable Network Constructions:}

Evaluate the attention on associated work which reflects the deployment buildings or application orientated results for cellular middle network. Since SDN, Soft mobile [8], Mobile Flow [9], Soft Mow [11] and SAMA [10] follow the idea of software defined networking at the movable essential community through changing the community purposes with software defined networking checkers and controls which was used to interrelate among the Radio Access Network and Outside Packet Networks. Mention [12] gives a qualitative conversation toward the benefits in addition disadvantages of using software defined networking for cell networks. Kemp etal. [13] current software defined networking center community structural design with additions to the Open Flow protocol to put into effect GPRS Tunneling Protocol to summarize customers' movement within the center community. SDMA [14] and Traffic Jam are proposed for a central network structural design primarily built on software defined networking with a focal point taking place consumer mobility administration the use of Open Flow. Together discuss that a software defined networking flexibility controller be able to enhance the central network assist on behalf of cellular operators. Additional route remains obtainable [15]. Awareness at the domain, e.g., person facts subways and accusing outlines that prerequisites to be gathered and replaced among software defined networking controls which put into effect control capabilities of the cell central community. Alternative has organization of schemes has investigated virtualization network function structure for the cellular essential community. Author in [17], and in [18] talk over a central network structure which is completely produced from digital community purposes and organized on the cloud infrastructure. The work in [19] gives the idea of Software as a Service(Saas) for a virtualized central community. Writers discussed in [20] make the most the ideas of Network function virtualization and cloud computing to offer a virtualized middle network which follows cellular customers by means of they flow. Moreover, the paintings in [10] and [11] gift a Network function virtualization middle structural design that runs along a general legacy center network. The Network function virtualization central network in these suggestions 
is used for depositing purposes in case the legacy center network remains overloaded. All the suggested cellular central community structural design within the literature survey recall moreover a deployment completely constructed totally on Network function virtualization or software defined networking [7].

\section{B. Source Allocation Problems for Dimensioning}

The most important regions of demonstrating and optimization associated with the usage of Network function virtualization and software defined networking inside the cell middle network: (a) placement of software defined networking controllers and switches and (b) aid provision and site of This trouble has been added wherever in uses a basic force approach to locate the employment of $\mathrm{K}$ wide variety of regulators and the task of switches towards each checker concentrated on a minimal manage aircraft potential.
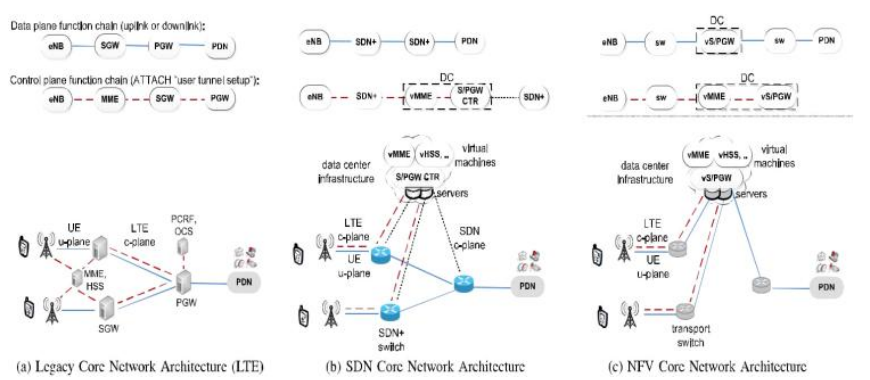

Fig. 1. Comparison of (a) Legacy Core Network

Architecture (LTE), (b) Software Defined Networking

Core Network Architecture and (c) Network function virtualization Core Network Architecture.

This diagram demonstrates the Logical User Data Plane,

Legacy Core Network Architecture(LTE) control plane and Software Defined Networking task chains of individually architecture with control plane.

The regulator assignment primarily built on a virtual strengthening heuristic was been suggested in through attention on manage aircraft potential as well as flexibility components. Salami also St-Hillarie offer a mathematical method on behalf of a most reliable regulator placement which reflects each regulator inactivity in addition to checkers capacity. The controller location that reduces the (CO) Control Overhead of distribution network info among disbursed regulators is suggested in allowing for the source distribution in addition to location of Virtualization Network Function 's, Gilbert etal, reveals a superior location for simulated center entries which cope with surprising traffic boom in situation of massive gathering occasions. Situation provides a scientific method in place of a gold standard assignment of virtual role chains. They recall constraints on the community potential in addition to asked expectancy on behalf of feature series. Reference suggests two procedures to implant system carrier chains through a goal of reducing the general implanting rate. Shi etal, Use device getting toward know strategies to discover a highest quality situation for virtualization network function`s specified records middle sources. The optimum vicinity responsive virtualization network function plotting is suggested in [17], which reduces the characteristic procedure as well as visitor's communication cost. In portable networks, the maximum efficiency on behalf of the network resources, that is hyperlink as well as node potential, was been suggested in [16], the wrapping of digital cellular central community features. Studying the present associated work on exhibiting in addition to maximum efficiency, here we able to examine that fashions that jointly take into account Network function virtualization and software defined networking are lacking. Moreover, just limited suggestions contain the particular tasks, procedures and necessities of the cell central community we goal at in this work. Additionally, there exist some suggestions which look into effect of data aircraft such that manipulate aircraft potential necessities. This trouble has been provided in [24] which uses an animal power manner to cope with discover the location K quantity of regulators and the venture of changes to individually regulator specializing in the base manage aircraft idleness. A controller state of affairs depending on a reproduced toughening experiential has been proposed in [25] with an emphasis on regulator aircraft inaction and versatility perspectives. Salami and St-Hillarie [26] supply a math-metical plan to a perfect controller association that thinks approximately each manipulate inertness and regulators load. The controller situation that limits the control overhead of sharing device statistics amongst dispersed controllers is proposed. Thinking about the asset designation and function of VNFs, Gebert et a showcase a great arrangement for virtual center doors that manage abrupt traffic increment if there should get up a prevalence of significant group occasions. Reference presents a systematic plan for a great state of affairs of digital capacity chains. They consider imperatives on the machine limit simply as mentioned inactiveness on behalf of potential chain. Situation suggests 2 calculations to implant device administration chain with a goal of limiting the general inserting fee [5]. Shi et all use AI methods to discover a great arrangement for virtualization network function's given server farm belongings. A perfect location conscious virtualization network function plotting is planned, which limits the capability getting ready in addition visitors broadcast rate. On behalf of transportable systems, the enhancement for system assets, that is connection in addition to hub limit, planned on behalf of the installing simulated flexible middle machine capacities.

Checking at the modern related writing on demonstrating and enhancement, we are able to see that fashions that collectively do not forget Network function virtualization and software defined networking are lacking. Moreover, just more than one tips fuse the point by means of factor capacities, obligations and prerequisites of the flexible middle machine as we cross for in our paintings. Moreover, there are just a couple of pointers that investigate the impact of the facts aircraft simply because the manipulate aircraft idleness stipulations. There are not any cutting-edge paintings, to our expertise, that is custom-made for versatile middle capacities and reflects the joint streamlining of VNF potential handcuffs just by means of software defined networking regulators as well as switches, this is the interesting point of our effort. 


\section{NETWORK ARCHITECTURE AND ANALYSIS USING NETWORK FUNCTION VIRTUALIZATION AND SOFTWARE DEFINED ETWORKING CENTRAL}

This segment, we talk over greater feature the following technology cell central network layout established on Network function virtualization and software defined networking. Moreover, we examine the influence of Network function virtualization and software defined networking on both statistics and the manage plane of cell essential network, that's altogether included within future maximum efficiency models.

\section{A. Network Architecture for Portable Central:}

LTE Mobile Core Network Construction: The cellular essential community, within the modern LTE well-known [9], Fig.(1a), contains of numerous community capabilities which put into effect distinct processes which might be wanted in place of a cell network. The essential network functions are categorized into two classes based on their perseverance: (a) capabilities that take care of the control aircraft best, consisting of the flexibility controlling entity otherwise the (HSS) Home-based Subscriber Server (b) capabilities that deal with the together statistics in addition to manage planes, consisting of the (SGW) Serving GateWay and the (PGW) Packet Data Network Gateway. Information aircraft tasks has implemented distinct motive handling for movable networks, i.e., GPRS Tunneling Protocol (GTP) mining the person information so that it will differentiate among the users and as a way to provide carrier first-rate lessons for every person. Additional information plane tasks consist of accounting and charging for the consumer statistics procedure. Manipulate plane features cope with the arrangement of the user's channels and flexibility controlling, that is monitoring location bring upto-date and resending of person channels. Furthermore, manipulate features take care of consumer authentication, subscription management and as get admission to manage. For more info, we confer with our previous work [12], in which we carried out an in depth evaluation of Long Term Evolution portable central community capabilities. In present day Long Term Evolution portable middle network, is legal, statistics in addition manage plane features be found out through means of devoted hardware that tools every expert characteristic. Affecting in direction of the next era 5G cellular core community, capabilities that simplest manage the manipulate plane, example, Mobility Management Entity will remain installed as a simulated community capability, that is, logical program, scheduled a data-cloud structure, that is, facts facilities. Though, concerning the features that hold both the facts in addition to manipulate planes, that is, Packet Data Network Gateway and Serving Gateway, here we think through the second awareness alternatives, both SDN primarily based or NFV based totally.

\section{B. Task Chains Investigation for Data Plane:}

The records level course inside the cellular center relies upon at the operator's conclusion for the awareness of each the PGW and SGW and tasks. In this situation the usage of Software Defined Network, as proven in Figure. 1-b, the legacy hardware capabilities might get substituted with the SDN + switches that is managed by way of the controls living for the data-cloud. In this manner the statistics plane itself would observe the equal task chains because the heritage community, that is, among the Software Defined Network+ changes. It additionally manner the information aircraft invisibility be influenced by only at the places of the Software Defined Network+ switches in addition to is decoupled from the area of the information middle infrastructure. The statistics plane traffic in cell networks may be demonstrated as single directional feature restraints, that is, upload or downlink.

\section{Task Chains Investigation using Control Plane:}

LTE manipulate plane techniques inside the transportable middle system include of various successive cycles among the machine capacities. For example, the ATTACH approach, allude to the 3GPP well known, consists of predominantly the Serving Gateway, Mobility Management Entity and Packet Data Network Gateway for the association of a client GPRS Tunneling Protocol Burrow. The ASSIGN method characterizes the manipulate packets traded in order to join a patron to the flexible device and arrangement its records plane GPRS Tunneling Protocol Burrow. It consists of three control cycles among the MME and the RAN, two manipulate emphases among the MME and the SGW and a couple of manage emphases among the SGW and PGW, in my opinion. Henceforth, the manipulate plane is required to be proven uniquely in assessment to how the manage plane is demonstrated in traditional Internet Protocol systems. Related work, as tested in Section Two-B, Example, fashions the manage aircraft capability restraints as single directional requests. This remained not coordinating the switch on the flexible middle gadget, where consecutive control emphases are required.

\section{Statement of Issue:}

The examination in the Sections (III-B) and (III-C), Here we should understand that Network function virtualization and software defined networking preparations for versatile center systems display tradeoffs as some distance as facts aircraft or control aircraft inertness, organize traffic and server farm property. Subsequently, novel development fashions are required to discover a most appropriate arranging and dimensioning for a portable middle device, that to acquire includes each SDN and NFV preparations, as far because the gadget burden fee and the server farm belongings value. The ideal middle system configuration entails an appropriate area for server farms and the right device break up among Network function virtualization and software defined networking that supports the normal extensive scope of site visitor's requests in 5-G. Also, best machine configuration wishes to guarantee the transportable middle gadget execution conditions, as a way as records aircraft and manipulate aircraft state of no activity. 


\section{NFV AND SDN BASED RESOURCE ALLOCATION MODELSAND MOBILE CORE DIMENSIONING}

The segment, three streamlining fashions are proposed for the correct value dimensioning of the flexible center gadget depending on both Network function virtualization and software defined networking ideas. In the present mathematical definition for the fashions and the utilized documentations for every one of the fashions. The streamlining representations are planned as Mixed Integer Linear Programs (MILP). All in all, the factor of the future fashions is to discover the ultimate measuring and asset designation of the center device that fulfills statistics plane and manipulate plane inertness necessities given a middle gadget network topology range of server farms. The fashions are applied to address a) the ideal association of the server farms, which host the portable Network function virtualization and software defined networking controllers, b) Suitable mapping of Virtual Network Function`s and switches to every datum recognition c) State and Variety of affairs of the unique cause software defined networking+ changes that execute the facts plane factors of the center device. The number one version specializes in the greatest device burden value, the subsequent model streamlines the server farm assets value, while the $1 / 3$ version is a Pareto ideal multipurpose model that outcomes in Pareto best fee for the machine burden and server farm belongings. The applied documentation for units, constraints and factors is introduced in Tables I to III, one after the other.

\section{A. Notation for Graph Model}

Middle net graph $\mathrm{G}(\mathrm{V}, \mathrm{E})$ is taken into consideration through a fixed of edges $\mathrm{E}$ and nodes $\mathrm{V}$. The center nodes are categorized as Serving Gateway node vs. $\in \mathrm{Vs} \subset \mathrm{V}$ and Packet data network Gateway nodes vice president $\in \mathrm{Vp} \subset$ V. We expect a Brownfield state of affairs in which an operator will select a region to install records middle (DC) wherein it already has installed node, as a result, facts middle nodes, that is, places, $\mathrm{C} \subseteq \mathrm{V}$. The set $\mathrm{D}$ holds glide demands in the middle community, where a flow demands $\mathrm{d}$ $=(\mathrm{vs}, \mathrm{vc}) \in \mathrm{D}$ signifies the asked bidirectional and nonsplittable data plane visitors float, that is, upload and downlink, among a Packet Data Network Gateway vs Serving Gateway node vc.
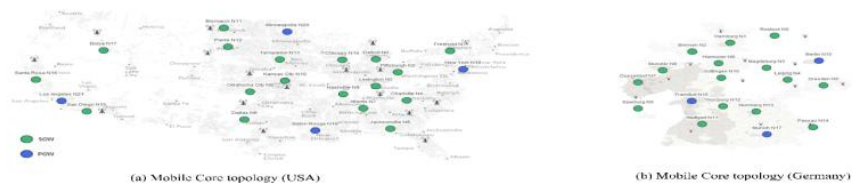

Fig. 2. Mobile Core Topologies, This Picture displays the positions of Serving Gateway`s (green) and Packet Data Network Gateway`s (blue). The analysis linked with the people is represented by the strength (grey) on the chart related.

Table 1

\begin{tabular}{|l|l|}
\hline notation & description \\
\hline $\mathrm{G}(\mathrm{V}, \mathrm{E})$ & V is node, $\mathrm{E}$ is edges of network graph \\
$\mathrm{C}$ & Group of nodes for data center $\mathrm{C} \underline{\mathrm{c} V}$ \\
$\mathrm{~V}^{\mathrm{S}}$ & Group of SGW nodes $\mathrm{V}^{\mathrm{S}} \underline{\mathrm{c}} \mathrm{V}$ \\
VP & Group of PGW nodes $\mathrm{V}^{\mathrm{P}} \underline{\underline{\mathrm{V}}}$ \\
\hline
\end{tabular}

Group of physical network edges
Group of traffic demand $d=\left(\mathrm{V}^{\mathrm{S}}, \mathrm{v}^{\mathrm{p}}\right) € \mathrm{~d}$
Group of data chain function for requested
$€ D, D C, c € C$
Group of control chain function for requested
$€ D, D C, c € C$

The information and controller planes of every ultimatum can be found out as Network function virtualization or software defined characteristic chains, correspondingly. Each call for, set Fd (c, d) incorporates the Network function virtualization and software defined facts aircraft characteristic chains of aultimatum $d \in D$ the use of a statistics center $\mathrm{c} \in \mathrm{C}$. Correspondingly, the set $\mathrm{Fc}(\mathrm{c}, \mathrm{d})$ includes the Network function virtualization and software defined regulator function chains of a call for $\mathrm{d}$ the usage of a statistics center $\mathrm{c} \in \mathrm{C}$. Concerning the Network function virtualization awareness of request $\mathrm{d}=$ (vs, vice chairman $)$, the statistics flat chain is described because the path traverse Serving Gateway node vs, the Virtual Network Function's installed at the records Packet Data Network Gateway node vice-chairman and middle nodes $\mathrm{c}$, at the same time as the switch aircraft is distinct as 3 instances the course between the Serving Gateway node vs Virtual Network Function set up.

\section{B. Cost Optimization representation for System Load}

In this model goes for advancing gadget value, it unearths the dimensioning and asset designation that offers an ideal system value. The version's price potential, what we name the gadget traffic burden or proper away gadget load, is characterized because the transmission capability idleness item. Along these lines, we should improve the system asset challenge, i.e., switch speed, however the presentation, dormancy, which would supply execution additions to the clients' know-how. Each potential series f utilizing server farm c for every hobby, the device burden figured because the stated switch velocity by using the hobby $\mathrm{r}(\mathrm{d})$ duplicated by means of the idleness at the capacity chain 
Table 2

\begin{tabular}{|c|c|}
\hline notation & description \\
\hline$\delta(\mathrm{c})$ & $\begin{array}{l}\text { Binary variable }=1 \text { if } \mathrm{DC} \text { is situated at } \mathrm{c} € \mathrm{C}, 0 \text { or } \\
\text { else. }\end{array}$ \\
\hline$\delta\left(\mathrm{c}, \mathrm{f}^{\mathrm{d}}, \mathrm{d}\right)$ & \\
\hline$\sigma^{\mathrm{d}}(\mathrm{c})$ & $\begin{array}{l}\text { Binary variable }=1 \text { if data plane of required } € D \text { is } \\
\text { chosen as a function } \\
\text { series } \int^{d} € f^{d} \text {, either } S D N \text { or } N F V \text {, using } D C, c € C \text {, } \\
0 \text { otherwise. }\end{array}$ \\
\hline \multirow[t]{3}{*}{$\begin{array}{l}\sigma^{c}(\mathrm{c}) \\
\mu(\mathrm{c})\end{array}$} & $\begin{array}{l}\text { Integer variable denoting number of center } \\
\text { required for control plane function chains at DC, } \\
\text { c } € C \text {. }\end{array}$ \\
\hline & $\begin{array}{l}\text { digit variable denote number of center essential } \\
\text { for control plane function chains at DC, c } € C \text {. }\end{array}$ \\
\hline & $\begin{array}{l}\text { digit variable denote the total number of servers } \\
\text { required for together data and control plane at } \\
\text { DC,c €C. }\end{array}$ \\
\hline
\end{tabular}

\section{TRADE-OFF's ASSESSMENT}

\section{A. Assessment System and Constraints:}

1) Frame-work: Here we use a Java frame-work for the development and the implementation of the three planned optimization fashions in segment(IV). Java frame-work is started to study the generating data plane traffic-demands and graph topology. Also additionally create the one-of-akind Network function virtualization and software defined networking task chains, as discuss in the Segment(III-B) and Segment(III-C), in which it computes the related constraints, i.e., community load, information center assets, records in addition to control aircraft latency. The framework makes use of Gurobi because the linear reduction solver for the applied model. At Last, the framework is used to calculate select constraints and attribute of the solution and paperwork ensuing Network function virtualization and software defined networking cellular center network system.

2) Topologies for Mobile Core: Cellular center community topology meant for America based at LTE insurance plan [12], which relates through the populace spreading and consider the positions of the Internet substitute points (I x P) [19], since explained in figure(2a). U.S.A Topology contains four Packet Data Network Gateway's in addition to eighteen Serving Gateway`s using complete twenty-two nodes, that is, ability facts middle positions. On behalf of assessment, here we utilize alternative cellular center system topology designed for Germany which has three Packet Data Network Gateway`s in addition to fifteen Serving Gateway's by means of a total eighteen nodes, proven in figure(2b). within these two topologies, every Serving Gateway node remains connected to the geologically nearby Packet Data Network Gateway node, correspondingly.

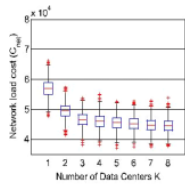

(a) Network load (optimals
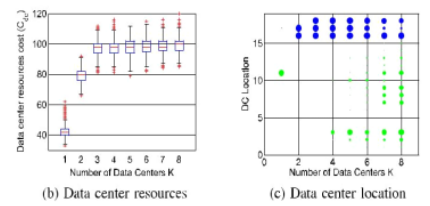

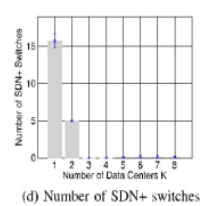

Fig. 3. A balance achieved between the network load price purpose in place of German topology, control potential $=\mathbf{5 0} \mathrm{ms}$ as well as data potential $=\mathbf{5} \mathrm{ms}$
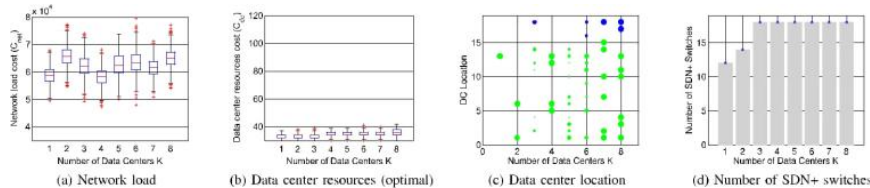

Fig. 4. A balance achieved between the data center resources price purpose in place of German topology, control potential $=50 \mathrm{~ms}$ and data potential $=5 \mathrm{~ms}$.

3) Control Plane in addition Data Traffic Demands: To assess the mobile core community dimensioning-price regarding to anticipated visitors enhance and the traffic dynamics brought by using Network function virtualization and software defined networking, we do not forget random site visitors requests for every information plane call for. The needs linking every Serving Gateway in addition to its adjacent Packet Data Network Gateway are regularly circulated among ten Gbps and fifty Gbps. The Control in addition to software defined networking visitors, we have got taken into consideration an arbitrary manipulate traffic fraction between $10 \%$ and $30 \%$ of the information traffic call for, which represent traditional LTE manage hundreds and revolutionary manipulate loads, e.g., gadget type verbal exchange. Visitor's expectations are projections from the estimated control plane and Data plane traffic enhance inside the subsequent generation 5-G community [1], for the numerical proof, the prototypes making more effective are evaluated for various runs up to a ninety-five $\%$ assurance is reached for the optimization result or at least for 30 runs.

\section{MULTI-OBJECTIVE MODELASSESSMENT \& RESULTS}

\section{A. Pareto Optimum Multi-Objective Model Gain:}

As we described in Segment (IV-D), multi goal technique repeats above unique weights $\lambda$ on behalf of every independent extending among $[0,1]$. Additional terms, that one travel through the answer space start as a result of way fixing 1 unmarried object, then shifting to remedy together targets concurrently, and stops subsequent tosolve the other single goal, hence producing the Pareto frontier among the two targets. For every weight $\lambda$, the setup is again recurringamong random varying traffic requests till the $95 \%$ fact come to or possibly by way of thirty runs. figure (7) represents Pareto desert among the device burden price and the server farm assets fee, for U.S. Topology and specified an information idleness prerequisite of $5 \mathrm{~ms}$ and a manipulate state of no activity necessity of fifty $\mathrm{ms}$. The assessment is surveyed for numerous server farms $K=(1$, four, 8). We may want to see that for a solitary unified server farm $K=1$, there isn't enough level of possibility to investigate the association space and give a parity or change off between the device burden price and server farm assets price. This is since with a focused server farm, the regions that satisfy each the information also control aircraft dormancy 
requirements are very restricted.



(a) Number of data centers $K=1$

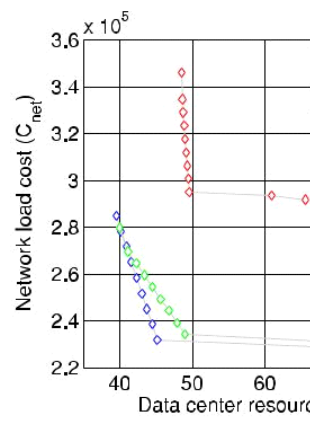

(b) Number of data
Fig. 5. Pareto frontier for data center resources cost $\left(C_{d c}\right)$ and network load cost $\left(C_{n e t}\right)$ for the U.S. topology, estimate the results of the Data Center Positions preselection and by way of optimal multiobjective model and with random Data Center Positions

Considering an appropriated server farm basis with $\mathrm{K}=4$, greater Pareto preparations contribution change offs among the 2 locations may be watched. For occasion the Pareto association at $\lambda=0.7$, the machine burden cost have just an above of three $\%$ contrasted with its ideal arrangement at $\lambda=$ 1 , at the same time as the server farm property cost outcomes in an overhead of four\% contrasted with its perfect arrangement at $\lambda=0$. Thinking approximately extraappropriated server farms at $\mathrm{K}=8$, we should see that may be extra level of possibility to cowl a larger arrangement space. For example, thinking about the Pareto arrangement at $\lambda=0$.Eight, the system burden fee has an above of five $\%$ contrasted with its perfect association, while the server farm assets witness a diffusion of $21 \%$ contrasted with its ideal association. It merits referencing that an administrator ought to cross for an exchange Pareto association relying upon the cost traits for every one of the gadget site visitor's load and the server farm property. All in all, the Pareto wasteland demonstrates the advantage of discovering arrangements that could not be efficaciously located through discretionary hundreds to every target inside the multi-target paintings. This offers administrators the likelihood to discover the appropriate device that harmonies between the machine burden price and server farm belongings fee.

B. Data Center Positions Pre-selection for the MultiObjective Optimization Model

We look at the assessment of our proposition of statistics middle regions pre-desire for the multi-target model as clarified in Segment (IV)-E. The pre-chosen server farm places are a mix of the subsequent regions from the preparations of the unmarried goal models, that is, prepare burden value model and server farm assets fee model. Give us a chance to do not forget a version with diverse server farms $K=4$. The association of the gadget burden price model, with four information centers, offers four ideal server farm regions that restriction the device burden price. Also, 4 ideal server farm areas are given by way of explaining the server farm assets value version with four server farms.

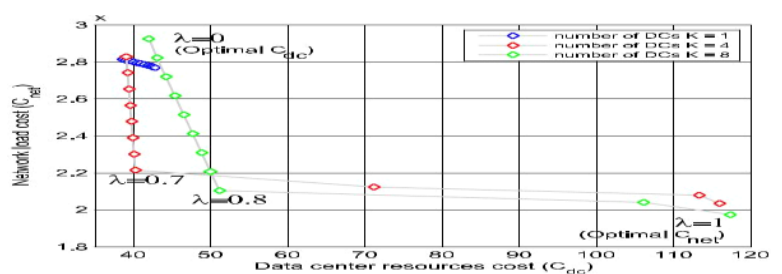

Fig. 6. Pareto cutting edge for the Network Load cost $\left(C_{n e t}\right)$ and Data Center Resources cost $\left(C_{d c}\right)$, cracking the Pareto Optimal Multi-Objective Model at number of data centers $K=(1,4,8)$ for the U.S. Topology

Two server farm regions are selected subjectively from the given preparations of each single target, in my opinion. The pre-chosen server farm regions structure the data established to the Multi-goal advancement model. Reminder that if there need to rise up an prevalence of a delivered together server farm $K=1$, a self-assertive vicinity a few of the coming about server farm regions from the answer of the two single locations is prechosen
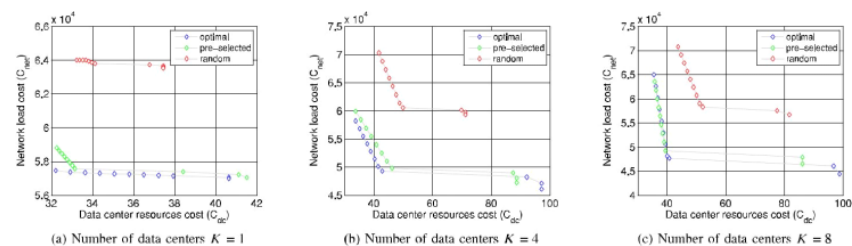

Fig. 7. Pareto cutting edge used for the (DCR)Data Center Resources price $\left(C_{d c}\right)$ and Network Load Cost $\left(C_{n e t}\right)$ for the German topology, relating the results of the

Data Center positions preselection with best multiobjective model and with random data center positions

The optimality gap through PreSelection in figure (6) and figure (7), Outline the Pareto boondocks assessment on behalf of the ideal multi-target version contrasted with the multi-target model using pre-choice for U.S. moreover, German-topology, respectively. Here we additionally examine the projected pre-determination, in mild of the preparations of the unmarried destinations, to an abnormal pre-desire. The arbitrary pre-willpower speaks to the state of affairs in which administrators as of now have fixed regions for the server farms and is settling the multi-target version for the given areas. The optimality hollow is the distinction among the 3 assessment instances at individual Pareto arrangement. Our team investigate optimality gap next to variety of server farms $\mathrm{K}=$ (one, four, eight) so as to analyze the effect of bringing together or dispersing the server farm framework

\section{CONCLUSION}

In this, we advise three improvement fashions that pass for finding an appropriate dimensioning and watching for a portable middle device depending on Network function virtualization and software defined networking, as far as gadget burden fee and server farm belongings price. The proposed fashions bring about the best association of server farms and the perfect transportable center device cut up 
amongst Network function virtualization and software defined networking. A wide assessment has been exhibited looking on the future models as a ways as the machine burden price and the server farm belongings value. Exchange offs among the single goal models could be watched, regarding the price factors simply as server farm regions. The multi-goal version outcomes in Pareto ideal arrangements wherein a harmony among the two price elements can be completed. Moreover, knowledge the multigoal version with the proposed server farm regions predesire has appeared vital development to the run time while maintaining a negligible hollow contrasted with the best Pareto preparations. For future paintings, greater fee factors can be taken into consideration for the streamlining fashions, for an instance, the rate of the software defined networking Plus controls or the among server farm joins. out to self-assertive locations on the middle device topology, i.e., no longer indistinguishable regions from the diagram hubs. Besides, the difficulties of the joint Concurrence of Network function virtualization and software defined networking flexible center capacities have to be tested, e.g., coordination and country dissemination. Moreover, a heterogeneous get admission to gadget may be displayed to speak to progressively sensible diagrams like USE-CASES designed on behalf of directors.

\section{REFERENCES}

1. Arsany Basta , Andreas Blenk, Klaus Hoffmann "Towards a Cost Optimal Design for a 5G Mobile Core Network based on SDN and NFV". IEEE trans. On network and service management, special issue on advances in management of softwarized networks, Page No's. 1-14.

2. Mobile and wireless communications Enablers for the Twenty-twenty Information Society (METIS), "Final report on architecturer (Deliverable D6.4)," Feb. 2015, https://www.metis2020.com/wp-content/ uploads/deliverables/METIS-D6.4-v2.pdf.

3. Final Report on Architectures (Deliverable D6.4), Mobile Wireless Communications. Enablers Twenty-Twenty Inf. Soc., Boston, MA, USA, Feb. 2015. [Online]. Available: tps://www.metis-2020.com/wpcontent/uploads/deliverables/METIS-D6.4-v2.pdf

4. Bo Han, Vijay Gopalakrishnan, Lusheng Ji, and Seungjoon Lee "Network Function Virtualization: Challenges and Opportunities for Innovations", IEEE Communications Magazine, feb-2015.

5. P.KumaraSwamy, Dr.C.V.GuruRao "A New Key Authentication Scheme for Cryptosystems Based on Discrete Logarithms" Journal of Innovation in Computer Science and Engineering, JUL, 2015,Vol 5 Issue 1, Page No's. 42-47

6. GowdiperuSucharitha , SrinivasAluvala, DeepikaVodnala , NagendarYamsani "A Survey on Mobile Ad-hoc Social Networks for Efficient Data Query in Intermittently Connected System" International Journal of Innovative Research in Computer and Communication Engineering, Vol. 3, Issue 11, November 2015, Page No's. 11831-11834

7. M.Rajesh, P.KumaraSwamy "SECURE MESSAGE AUTHENTICATION IN PERVASIVE COMPUTING" International Journal For Technological Research In Engineering, Vol. 3, Issue 4, December 2015, Page No's. 840843.

8.

9. Dhana Lakshmi, Dr.R.VijayaPrakash "A survey on Secure and Privacy Preserving Information Brokerage System The association of server farms regions might be stretched

Against Intruders" International Journal of Computer Applications and Engineering Technology, Vol. 4, Issue 1, January 2015, Page No's. 309-314.

10. Arijit Banerjee, Xu Chen, Jeffrey Erman, Vijay Gopalakrishnan2, Seungjoon Lee and Jacobus Van der Merwe, "MOCA: A lightweight mobile cloud offloading architecture," in Proc. 8th ACM Int, USA, 2013, Page No. $11-16$.

11. Junguk Cho, Binh Nguyen, Arijit Banerjee, Robert Ricci, Jacobus Van der Merwe, Kirk Webb, "SMORE: Softwaredefined networking mobile offloading architecture," in Proc. 4th Workshop All Things Cell, 2014, Page No. 21-26.

12. Arsany Basta, Wolfgang Kellerer, Marco Hoffmann, Klaus Hoffmann, and Ernst-Dieter Schmidt, "A virtual SDN-enabled LTE EPC architecture: A case study for S-/P-gateways functions," in Proc. IEEE, Trento, Italy, 2013, Page No. 1-7.

13. Brandon Heller, Rob Sherwood, and Nick McKeown, "The controller placement problem," in Proc. ACM, NY, USA2012, Page No. 7-12.

14. S. Lange et al., "Heuristic approaches to the controller placement problem in large scale SDN networks," IEEE Trans. Netw. Service Manag., vol. 12, no. 1, pp. 4-17, Mar. 2015.

15. A. Sallahi and M. St-Hilaire, "Optimal model for the controller place-ment problem in software defined networks," IEEE Commun. Lett., vol. 19, no. 1, pp. 30-33, Jan. 2015.

16. T. Mahmoodi and S. Seetharaman, "Traffic jam: Handling the increasing volume of mobile data traffic," IEEE Veh. Technol. Mag., vol. 9, no. 3,56-62, Sep. 2014

17. H. Lindholm, L. Osmani, H. Flinck, S. Tarkoma, and A. Rao, "State space analysis to refactor the mobile core," in Proc. 5th Workshop AllThings Cell. Oper. Appl. Challenges, 2015, pp. 31-36.

18. V.-G. Nguyen, T.-X. Do, and Y. Kim, "SDN and virtualization-based LTE mobile network architectures: A comprehensive survey," WirelessPers. Commun., vol. 86, no. 3, pp. 1401-1438, 2016.

19. H. Baba, M. Matsumoto, and K. Noritake, "Lightweight virtualized evolved packet core architecture for future mobile communication," in Proc. IEEE Wireless Commun. Netw. Conf. (WCNC), New Orleans, LA,USA, 2015, pp. 1811-1816.

20. W. Kiess, X. An, and S. Beker, "Software-as-a-service for the virtu-alization of mobile network gateways," in Proc. IEEE Glob.Commun.Conf. (GLOBECOM), San Diego, CA.

21. K. Wang et al., "MobiScud: A fast moving personal cloud in the mobile network," in Proc. 5th Workshop All Things Cell. Oper. Appl.Challenges, 2015, pp. 19-24.

\section{AUTHOR's PROFILE}

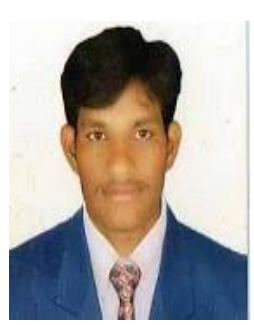

Vijay Kumar Bura received his Bachelors Degree (B.Tech) in Computer Science Information Technology from JNTUH in 2006 and Master's degree (M.Tech) in Software Engineering form Jawaharlal Nehru Techno-logical University, Hyderabad, Telangana, India in 2011. He worked as Software Engineer at ITP Software India Private Limited, Hyderabad for 2 years. He developed various web applications for different clients. He worked as Asst. Prof. in the Dept. of IT, SVS Institute of Technology, Warangal for 2 years. Presently he is working as Assistant Professor in the

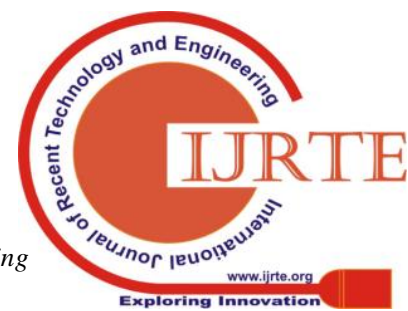


Department of Computer Science and Engineering, S R Engineering College (Autonomous), Warangal Telangana, India. As a mentor, he represented a team to participate in CISCO IoT Hackathon 2017 held at Trident Group of Institutions, Orissa, The team idea was selected for "Best Jury Award" and secured RUNNER UP position. https://www.facebook.com/Cisco.India.IoT.Hackathon/

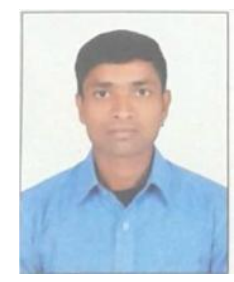

Yerrolla Chanti received Master's degree in Computer Science and Engineering in 2016 from Jawaharlal Nehru Technological University, Hyderabad, India. He has the teaching experience of 3 years. Currently, he is working as Associate Professor in Computer Science and Engineering Department at S R Engineering College, Warangal, India. His research areas include Networking, Big Data Analytics.

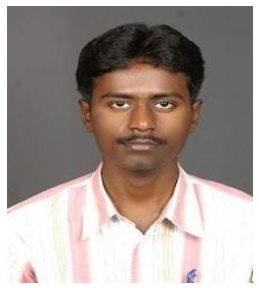

Nagendar Yamsani received Master's degree in Computer Science and Engineering in 2009 from Jawaharlal Nehru Technological University, Hyderabad, India. He is Pursuing Ph.D. degree in the stream of IoT in Computer Science and Engineering at $\mathrm{K} \mathrm{L}$ University, Guntur, Andhra Pradesh, India. He has 9 years of teaching experience. Currently he is working Assistant Professor in the Department of Computer Science and Engineering in S R Engineering College (Autonomous), Telangana, India and Coordinator, S R Research \& Development Center. $\mathrm{He}$ has published Eighteen International Journals and Three International Conference Papers. His research areas include Networks Security, Automata and Data Mining. He is a Life Member of Indian Society for Technical Education (ISTE).

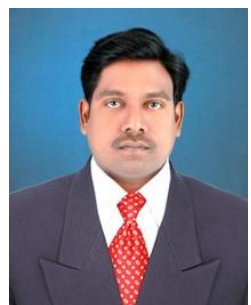

Srinivas Aluvala received his Masters degree in Computer Science and Engineering (M.Tech) form Jawaharlal Nehru Technological University, Hyderabad, Telangana, India in 2010. He is Pursuing Ph.D. degree in the stream of Mobile ad-hoc networks in Computer Science and Engineering at KL University, Guntur, and Andhra Pradesh, India. He has 10 years of teaching experience. Currently he is working as Assistant Professor and heading the Department of Computer Science and Engineering in SR Engineering College (Autonomous), Telangana, India. He is executing a research project titled "Development of Adhesive Tactile Walking Surface Indicator for Elderly and Visually Impaired People".

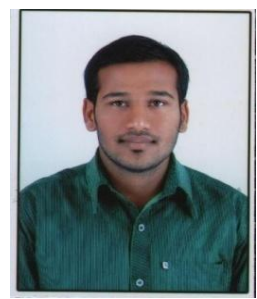

Bandi Bhaskar is an Assistant Professor of Computer Science and Engineering Department, S R Engineering College, Warangal. He received M.Tech [Software Engineering] from KITS, Warangal in the year $2013 \&$ he received B.Tech
[Information Technology] from Ganapathy Engineering College, Warangal affiliated to JNTU, Hyderabad in the year 2011. His area of interest includes Design Patterns, Adhoc Sensor Networks, Advance Data Bases and all Current trends and Techniques. 